\title{
Annexe 2 : Loi des 11-12 juin 1880. Chemins de fer d'intérêt local, Tramways
}

\section{(2) OpenEdition}

\section{Journals}

Édition électronique

URL : https://journals.openedition.org/rhcf/2096

DOI : 10.4000/rhcf.2096

\section{Éditeur}

Rails \& histoire

\section{Édition imprimée}

Date de publication : 2 mai 2002

Pagination : 412-420

ISBN : 00996-9403

ISSN : 0996-9403

Référence électronique

«Annexe 2 : Loi des 11-12 juin 1880. Chemins de fer d'intérêt local, Tramways », Revue d'histoire des chemins de fer [En ligne], 24-25 | 2002, mis en ligne le 28 avril 2015, consulté le 22 avril 2022. URL http://journals.openedition.org/rhcf/2096 ; DOI : https://doi.org/10.4000/rhcf.2096

Ce document a été généré automatiquement le 22 avril 2022

Tous droits réservés 


\section{Annexe 2 : Loi des 11-12 juin 1880. Chemins de fer d'intérêt local, Tramways}

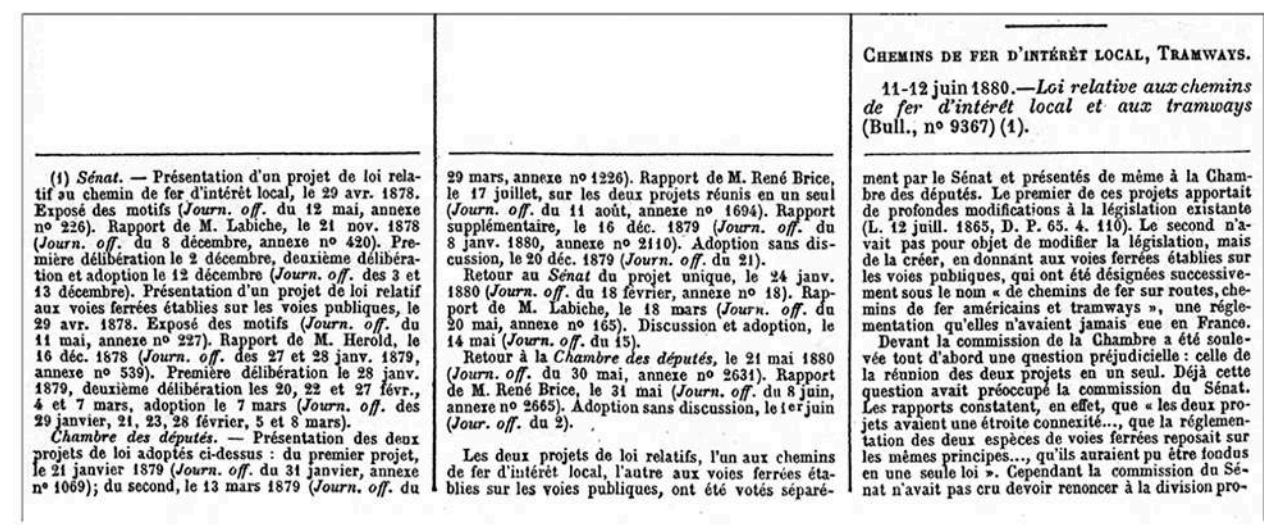

Dalloz, Lois, décrets et actes législatifs, 1881, 4e partie, fac-simile des pages 21-24 
Gнap. 1er. - Chemins de fer d'intérét local.

Art. 1er. L'établissement des chemins de par les communes, avec ou sans le concours
des propriétaires intéressés, est soumis aux ispositions suivantes (1).

2. S'il s'agit de chemins à établir par un plusieurs communes, le conseil général arrête, après instruction préalable par le prémins, le mode et les conditions de leur positions nécessaires pour en assurer l'exploitation, en se conformant aux clauses et conditions du cahier des charges type approuve par le conseil d'Etat, sauf les modivention et la loi derapprobation par la conSi la ligne doit s'etendre sur

partements, il y aura lieu à l'application des art. 89 et 90 de la loi du 10 aout 1871. a établir par chemins de fer d'intérêt local toire les attributions confées sur son terrinéral par le paragraphe 1or du présent article seront exercées parle conseil municipa dans les memes conditions et sans qu'il soi Let de lapprobation du prefet.

local departementaux ous de fer d'intér arrêtés, sont soumis à l'examen du conse général des ponts et chaussées et du conseil d'Etat. Si le projet a été arrêté par un conse conseil général accompagné de l'avis du L'utilité publiqu

tion est autorisée par une loi (2)

3. L'autorisation obtenue, s'il s'agit d'un chemin de fer concédé par le conseil génégénieur en chef du département avis de l'inprojets d'exécution au conseil général, qui statue définitivement. Néanmoins, dans les deux mois qui suí-
vent la délibération, le ministre des travaux publics, sur la proposition du préfet, peut, pres avoir pris lavis du conseil general des

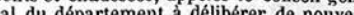
sur lesdits projets.

Si la ligne doit s'étendre sur plusieurs départements, et s'il y a désaccord entre les conseils generaux, le ministre statue. S'il s'agit d'un chemin concédé par un par le conseil général, aux termes du pararaphe 10r du présent article, appartiennent au conseil municipal, dont la délibération Si un chemin de fer d'intérétet. morunter le sol "d"une voie publique projets' d'exécution sont précédés de renquêet prevue par l'art. 29 de la présente loi. Dans ce cas, sont egalement applicables Les projets de dstail des our approuvés par le préfet, sur l'avis de l'ingénieur en chef (3).

4. Lacte de concession détermine les droits e peage et les prix de transport gue le concoute la durée de sa concession. pendant 5. Les taxes perçues dans les limites du maximum fixé par le cahier des charges sont homologuees par le ministre des traaux publics, dans le cas oú la ligne s'etena de tarits communs à plusieurs lignes. Elles ont homologuées par le préfet dans los au-

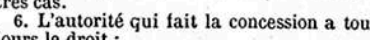
jours le droit

s'embrancher sur d'autres voies ferrées y raccorder: $2^{\circ}$ D'accorder à ces entreprises nouvelles, ficente payement des droits de peag

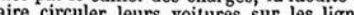
concédées:

30 De racheter la concession aux conditions qui seront fixées par le cahier des charges ;
40 De supprimer ou de modifier une partie du tracé lorsque la nécessité en aura éte

Dans ces deux derniers cas, si les droits un accord préalable ou par un réses par tabli soit par le cahier des charges, soit par une convention posterieure, l'indemnité qui peut lui etre due est liquidée par une au paragraphe 3 de l'art. 11 de la présente 7. Le cahier des charges détermine : ionnes droits et les obligations du conces20 Les droits et les obligations sionnaire à l'expiration de la concession: $3^{\circ}$ Les cas dans lesquels l'inexécution des conditions de la concession peut entrainer la mes aire déchu dre a legard a concesionLa déchéance est prononcée, dans tous les cas, par le ministre des travaux publics, sauf recours au conseil d'Etat par la voie

8. Aucune concession ne pourra faire obsons concurrentes, a moins de stipulation or A dans lacte de concession. 9. A l'expiration de la concession, le concessionnaibstitue a tous les droits du convent lui être remises en bon état d'entretien. Le cahier des charges règle les droits et les obligations du concessionnaire en ce qui concerne les autres objets mobiliers ou im10. Toute cession totale ou partielle de la concession, la fusion des concessions ou des administrations, tout changement de concesdirecte l' la sabstitution de l'exploitation ation des tarifs au-dessus da maximum fixé, ne pourront avoir lieu qu'en vertu d'un Cécret délibéré en conseil d'Etat, rendu sur l'avis conforme du conseil général, s'il s'agit

Dalloz, Lois, décrets et actes législatifs, 1881, 4 partie, fac-simile des pages 21-24
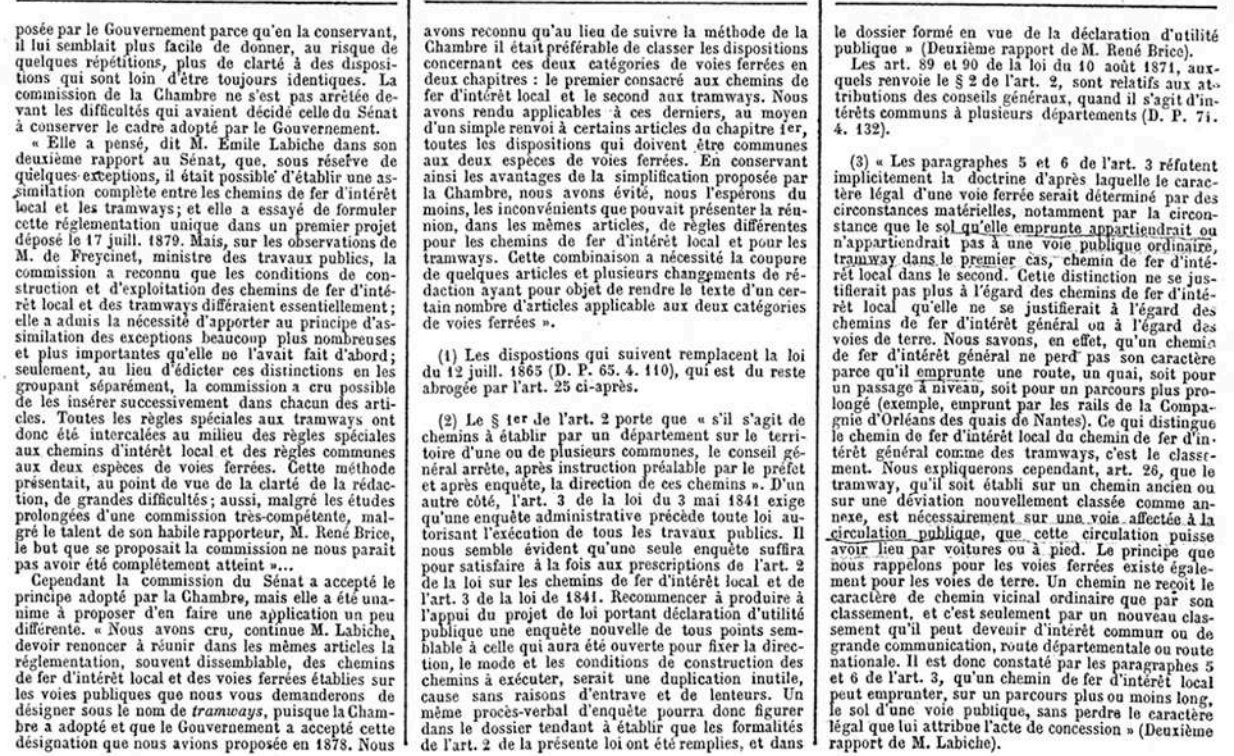

Dalloz, Lois, décrets et actes législatifs, 1881, 4e partie, fac-simile des pages 21-24 


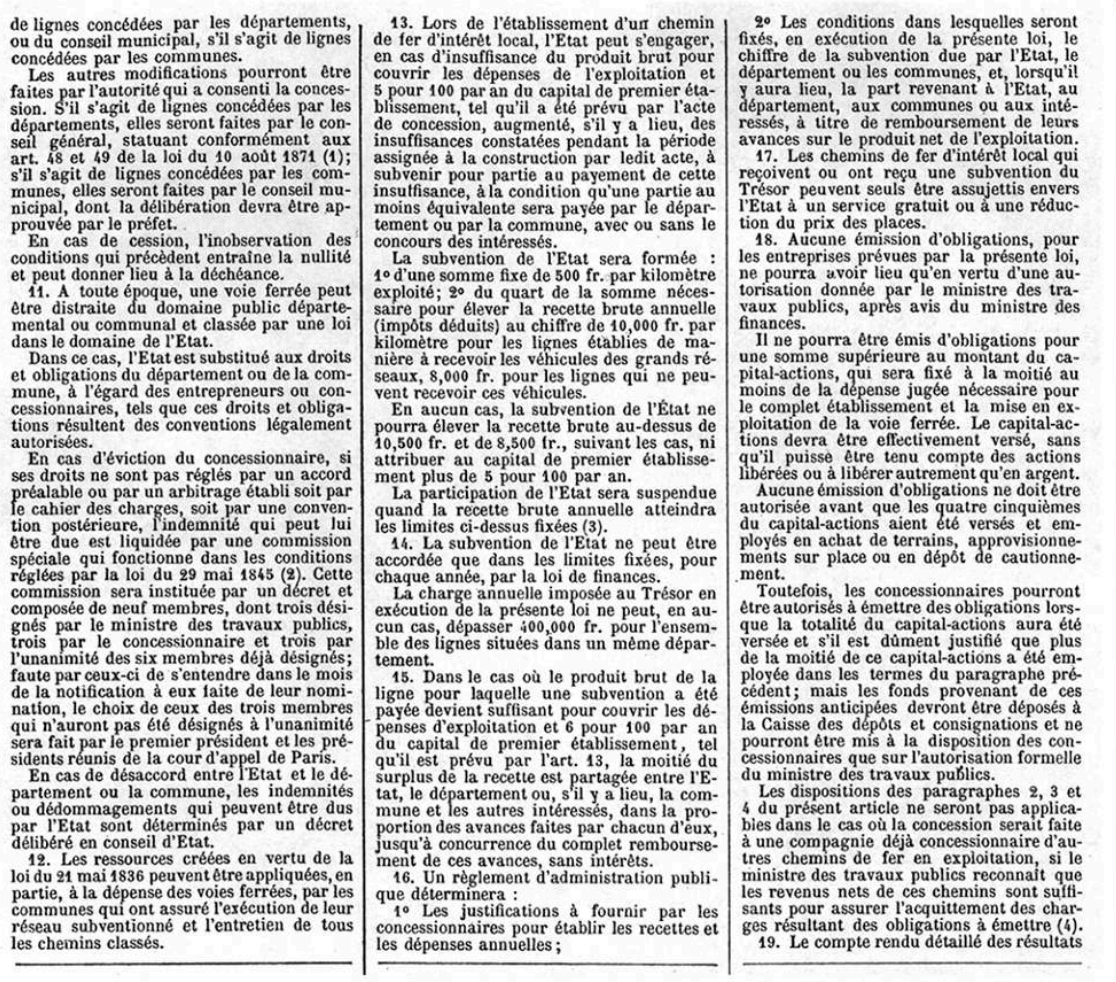

Dalloz, Lois, décrets et actes législatifs, 1881, 4e partie, fac-simile des pages 21-24

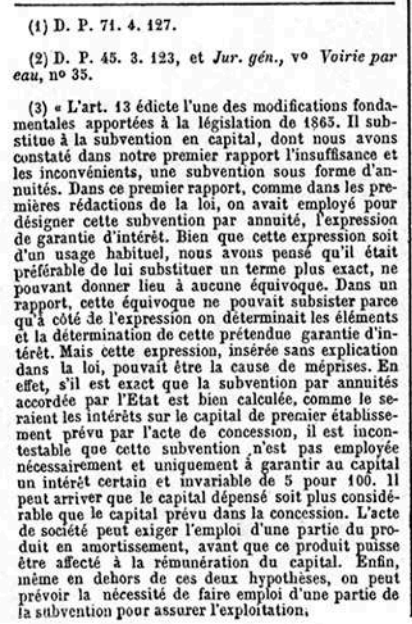

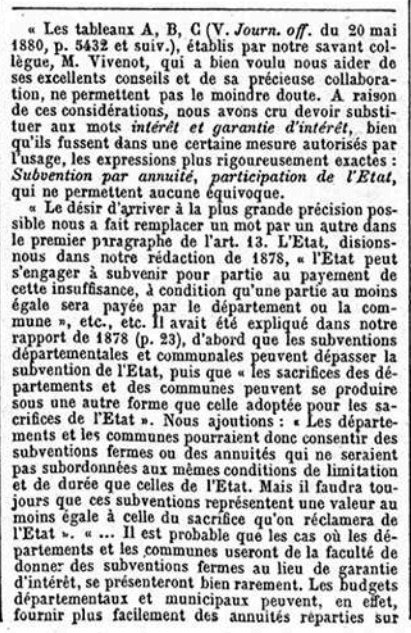

Dalloz, Lois, décrets et actes législatifs, 1881, 4e partie, fac-simile des pages 21-24 
de l'exploitation, comprenant les dépenses
d'établissement et d'exploitation et les re d'établissement et d'exploitation et les re-
cettes brutes, sera remis tous les trois mois, cettes brutes, sera remis tous les trois mois,
pour etre publie, au préfet, au president de pour commission départementale et au ministre des travaux publics.

Le modele des documents à fournir sera arreté par le ministre des travaux publics. loi du 15 juill. 1845 , sur la police des che mins de fer, le préfet peut dispenser de poser des clotures sur tout ðu partie de la voie rerrée, il peut également dispenser de po-
ser des barrières au croisement des chemin seu fréquentés.
pu croisement des chemin

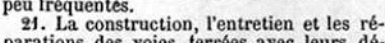
parations des voies terrées avec leurs dependances, lentretien du materiel et le ser-
vice de l'exploitation sont soumis au contrót et à la surveillance des préfets, sous l'autoLes frais de controle sont í la ch. concessionnaires. Ils seront réglés par le cahier des charges ou, a defant, par le préfet, sur ravis au conseil genéral, et a prouves par re ministre des travaux publics.
22. Les dispositions de lart. 20 de la présente loi sont également applicables aux
concessions de chemins de fer industrie concessions de chemins de fer industries
destinés à desservir des exploitations parti-

23. Sur la proposition des conseils géné hésion des concessionnaires, la substitution aux subventions en capital, promises en
exécution de l'art. 5 de la lai de 1865 , de la subvention en annuités stipulée par la conseil d'Etat étre autorisée en faveur lignes d'intérêt local actuellement déclarées dutilite publique et non encore exécutée. Ces lignes seront soumises des lors a to

loi. Il n'y aura pas lieu de renouveler les con-
cessions consenties ou les mesures d'instruetion accomplies avant la promulgation la présente loi, si toutes les formalités qu'elle prescrit ont été observées par avance.

24. fer d'intérét local, ainsi que les cahiers des charges annexes, ne seront passibles que

roit d'enregistrement fixe de 1 franc.
95. La loi du 12 juill. 1865 est abrogée. Chup. 2. - Tramways.

26. Il peut être établi sur les voies dédépartements ou des communes, des tramways ou voies ferrées a traction de chevaux ou de moteurs mécaniques. Ces voies ferrées, ainsi que les déviations accessoires construites en dehors du sol des routes et chemins et classees comme an-
nexes, sont soumises aux dispositions suivantes (1).

27. La concession est accordée par l'Etat lorsque la ligne doit être établie, en tout
ou en partie, sur une voie dépendant du domaine public de l'Etat.

Concession peut etre faite aux villes ou aux départen.

La concession est accordée par le conse général, au nom du département, lorsque a voie ferree, sans emprunter une roule partie, soit sur une route départementale, soit sur un chemin de grande communication ou d'intérét commun, ou aort s'étendre sur lerritoire de plusieurs compans. partements, ily aura lieu à l'application des art. 89 et 90 de la loi du 10 août 1871 . La concession est accordée par le conseil municipal lorsque la voie ferree est étabis entierement sur un chemin rural.

28. Le département peut accorder la concession a l'Etat ou a une commune, avec faculté de rétrocession; une commune peu agir de même à l'égard de l'Etat ou du dé29. Aucune concession ne peut être faite quanres une enquête dans les formes dé tion publique et dans laquelle les conseils généraux des départements et les conseils municipaux des communes dont la voie doit qu'il ne leur appartient entendus, lorssur la concession.

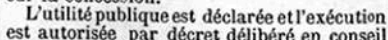
est autorisée par décret délibéré en conseil
d'Etat, sur le rapport du ministre des tra d'Etat, sur le rapport du ministre des tra-
vaux publics, aprés avis du ministre de l'intérieur (2). 30. Toute dérogation ou modification apportée aux clauses du cahier des charges type, approuvé par le conseil d'Etat, devra passés au sujet de la concession, lesquels au décret.

31. Lorsque, pour rétablissement d'un tramway, il y aura lieu a expropriation, nal, soit pour l'une des d'un chemin vicinal, soit pour l'une des déviations prévues
à lart. 26 de la présente loi, cette expropriation pourra étre opérée conformément lart. 16 de la loi du 21 mai 1836 , sur les
chemins vicinaux, et à lart. 2 de la lol chemins vicinaux, et à l'art. 2 de la loi
du 8 juin $1864(3)$. 32. Les projetsd'exécution sont approuvés par le ministre des travaux publics, lorsque la concession est accordée par l'Etat.
Les dispositions de l'art. 3 sont applicaLes dispositions de lart. 3 sont applica-
bles lorsque la concession est accordee par un département ou par une commune pa 33. Les taxes percues dans les limites du maximum fixé par l'acte de concessio sont homologuées par le ministre des travaux publics, dans. le cas oú la concession les autres cas. 34. Les concessionnaires de tramways ne sont pas soumis a limpot des prestations établi par l'art. 3 de la loi du 21 mai 1836

Dalloz, Lois, décrets et actes législatifs, 1881, 4 partie, fac-simile des pages 21-24
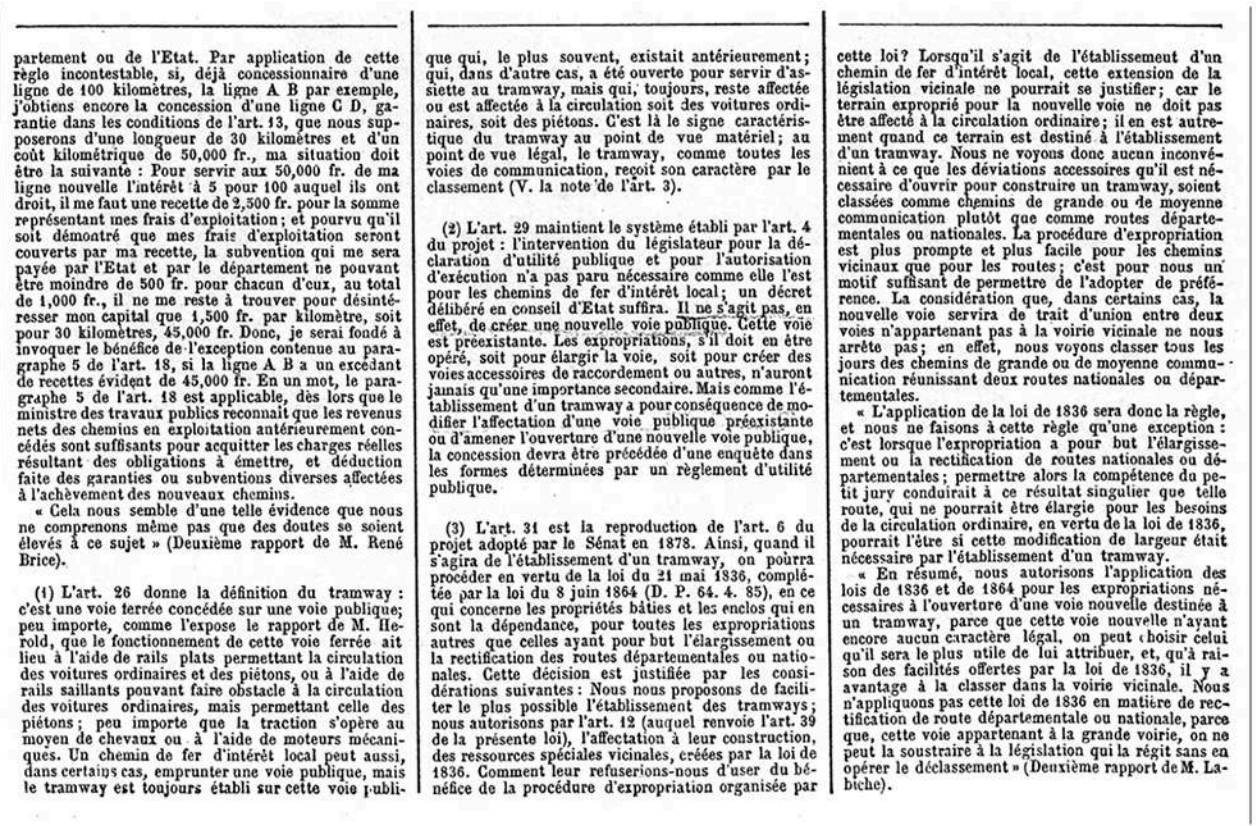

Dalloz, Lois, décrets et actes législatifs, 1881, 4 partie, fac-simile des pages 21-24 


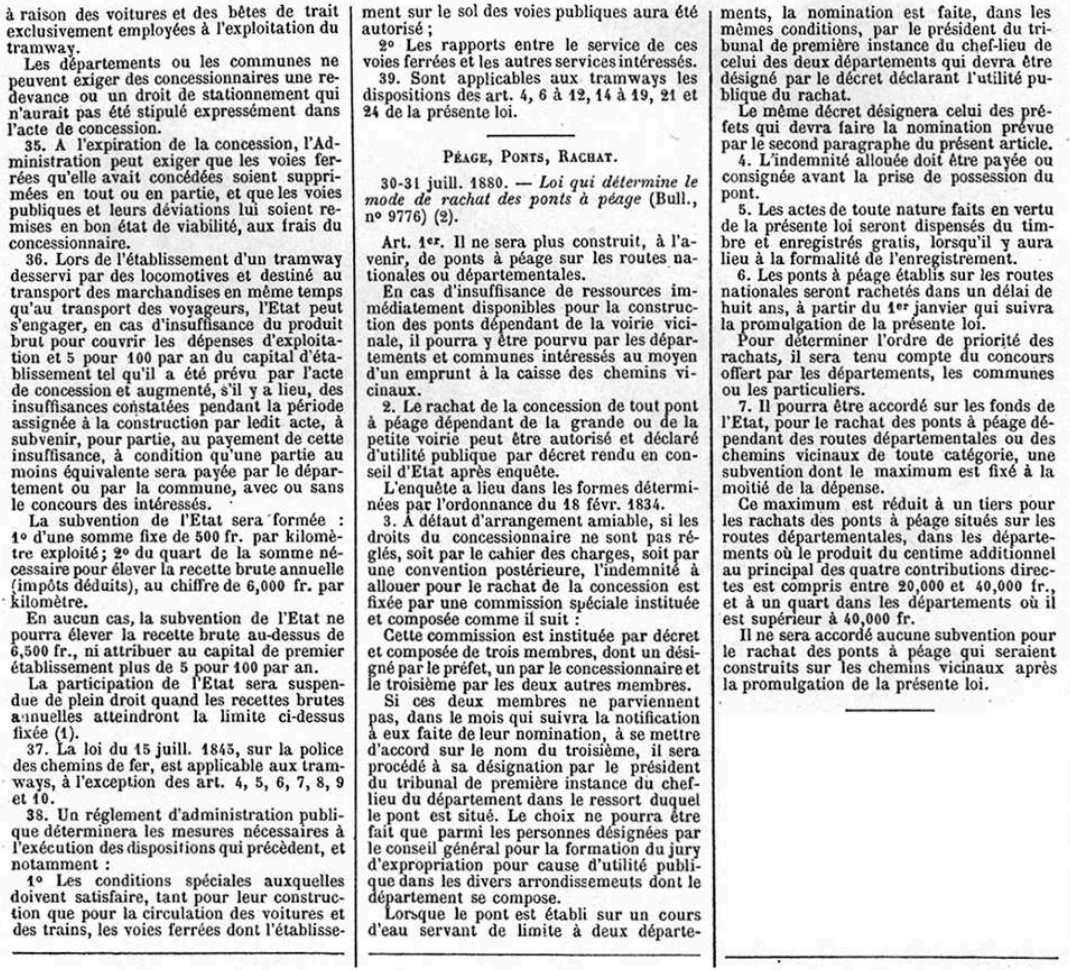

Dalloz, Lois, décrets et actes législatifs, 1881, 4e partie, fac-simile des pages 21-24

(1) V. l'art. 13 ci-dessus et la note.

(9) Sénat. - Présentation le 1er juill. 1879. Ex-
pose des motifs (Journ. off. da 15 juillet, annex

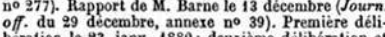

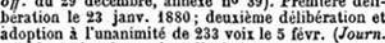
off. du 24 janvier et du 6 février). Chambre des députés. - Présentation le 18 mars
1880 (Journ. off du 22 avril, annexe no 2458 ). Proposition de loi présentée par Mi. de La Porte le 26 juill.
1879 (Journ. off. du 15 aout 1879, annexe no 1793 ) Rapport do M. Me La Porte le 13 mai 1880 (Journ. off.
des 28 mai et 5 juill. 1880 , annese no 2608). Délathion d'urgence, discussion et adoption le 29 juin

(Journ. off. du 30 ).
Retour au Sénat 1 g juill. 1880 (Journ. off. do
25 juillet, annexe no 448 ). Rapport de M. Barne et it juillet et 96 aongence le 10 juillet (Jour discussion a rananimité de 235 voix le 12 juill

(3) Sénat. - Présentation le 2 mars 1880. Expose
des motifs (Journ. off. du 18 mars, annexe no 89 . f. du 6 mai, anvere no 974). Premiere dition le 10 mai. Deuxieme délibération et adoption le $25 \mathrm{~m}$ Chambre des députés. - Présentation le 99 mat 1880 (Journ. off. du 12 juin, annexe no 2661 ). Rap.
port de M. Leroy, le 22 juin (Journ. off. du 9 juil let, annexe no 2781 ). Déclaration d'urgence et adop-
tion sans discussion le 29 juin (Journ. off. du 30 ).

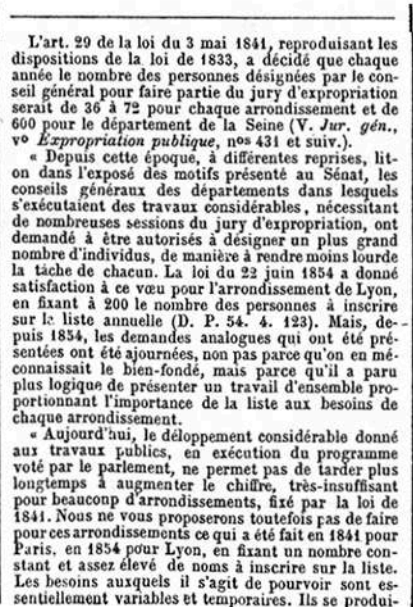

Paris, en 1854 pour Lyon, en fixant un nombre con-
stant et assez elevé de onoms a inscrire sur la liste.
Les besoins auxquels il s'agit de pourroir sont es-
sentiellement variables et temporaires. Ils se produi-

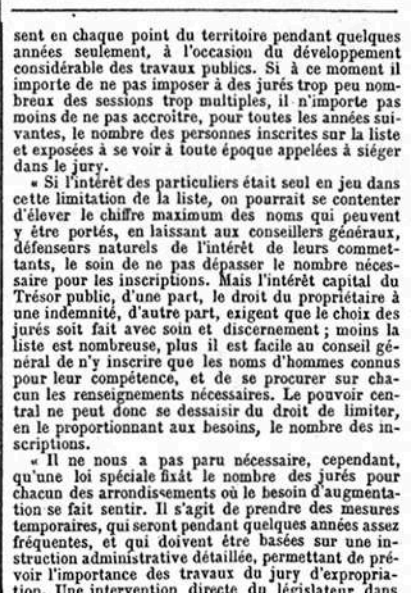

